\title{
Intrahepatic Cholestasis of pregnancy in the prenatal: case report
}

\begin{abstract}
Background: Intrahepatic cholestasis of pregnancy is a rare disease with uncommon clinical status, triggered by the gestational process and of important repercussions for pregnant women and babies.

Objective: report a case of intra hepatic cholestasis of pregnancy. Case: A 39-year-old female nurse, in the first pregnancy, at the 35th week of pregnancy, experienced intense full-body itching related to the allergic process associated with choluria, fecal acholia and jaundice. There was a suspected diagnosis of intrahepatic cholestasis of pregnancy, but after examination in an emergency department and also liver and kidney function tests, among others, the diagnosis was confirmed. A medicative drug treatment with ursodeoxycholicacid was performed to relieve the symptoms and dexamethasone was used for fetal maturation, as well as week lymonitoring of the conceptus vitality, with a favorable outcome.

Conclusion: Giventhe case, is remarkable the importance of obtainingan early diagnosis of Intrahepatic Cholestasis of Pregnancy, requiring experience and a critical and questioning look of the examiner during prenatal consultations. It also high lights the need for further studies on strategies aimed at facility at ing the earliest possible diagnosis and treatment in order to avoid fatal outcomes.
\end{abstract}

Keywords: Cholestasis Intrahepatic, pregnant women, prenatal care, early diagnosis, women's health
Volume 10 Issue 5 - 2019

Silas Santos Carvalho,' Bruno Rodrigues de Oliveira, ${ }^{2}$ Débora Oliveira de Matos, ${ }^{3}$ Aline Macedo Carvalho Freitas,' Bruna Gesteira Pimenta, ${ }^{3}$ Camila da Silva e Silva, ${ }^{4}$ Carine Andrade dos Santos, ${ }^{5}$ Carolina Santos Cerqueira, ${ }^{6}$ Jeane Silva Costa, ${ }^{3}$ Letícia Falcão de Carvalho,' Lorena Ramalho Galvão,' Luciana Bareto Carvalho, ${ }^{3}$ Michely Machado da Purificação, ${ }^{7}$ Polyanna de Lima Alves, ${ }^{3}$ Roberta de Almeida Soares, ${ }^{7}$ Tamara da Silva Souza Nogueira, ${ }^{4}$ Denise Angela Bacelar Soares, ${ }^{4}$ Kézia França de Jesus, ${ }^{1}$ Jayanne Moreira Carneirol

Universidade Estadual de Feira de Santana, Brasil 'Universidade Salvador, Feira de Santana, Brasil ${ }^{3}$ Faculdade Ateneu, Feira de Santana, Brasil ${ }^{4}$ Faculdade Anísio Teixeira, Feira de Santana, Brasil

${ }^{5}$ Faculdade Nobre, Feira de Santana, Brasil

'Universidade Estadual da Bahia, Salvador, Brasil

'Faculdade de Tecnologia e Ciências, Feira de Santana, Brasil

Correspondence: Silas Santos Carvalho, Departamento de Saúde, Universidade Estadual de Feira de Santana, Feira de Santana, Bahia, Brasil, Tel (75) 99996-7990,

Email ssc.macademico@hotmail.com

Received: September 21, 2019 | Published: September 30, 2019
Abbreviations: IHCP, Intrahepatic Cholestasis of pregnancy; GTO, glutamic transaminase oxalacetic; GT, glutamic transaminase; gamma GT, gamma glutamil transpeptidase; ANF, anti-nuclear factor

\section{Introduction}

Intrahepatic cholestasis of pregnancy (IHCP) is a rare disease triggered by the gestational process. Although its etiology is not fully known, environmental, geographical, nutritional, genetic and hormonal issues are identified as predisposing factors and hyperestrogenemia is an important factor in predisposed women. Its incidence worldwide is between 0,5 and $2,0 \%$ of all pregnancies, being more affected in women with multiple pregnancy and over 35 years of age..$^{1-3}$

Maternal prognosis is considered satisfactory, with symptoms resolved immediately after delivery or until 48 hours after, and worsening of liver function is rare compared to other liver diseases affected during pregnancy, and relapses may occur in the next pregnancies, especially if the patient remains pregnant, use of oral estrogen-based contraceptives and/or hormone replacement therapy. Puerperal hemorrhages may occur if there is no previous correction of vitamin $\mathrm{K}$ deficiency caused by malabsorption. ${ }^{2,4}$

Fetal prognosis is often associated with 27 -week premature birth, presence of meconium, fetal bradycardia, fetal distress, and death of the fetus. There are no concrete explanations for high morbidity and mortality, however, justified by serum bile acid increase. These are thought to contribute to contraction of the placental chorionic veins and the myometrium becomes more sensitive to the action of oxytocin and should therefore be treated as a high-risk pregnancy.

It is evident the scarcity of scientific production in Brazil that addresses this content, justified by the unequal distribution of new cases worldwide and the impossibility of carrying out clinical studies with ethically acceptable results, further favoring the lack of knowledge on this subject and making it difficult to attend, to this pregnant woman in ensuring the resolution of her complaints during prenatal care. ${ }^{5}$

Given the clinical importance that this disease gives to the pregnancy-puerperal process, this study aims to report the case of a nurse with Intrahepatic Cholestasis of pregnancy. The case reported and the publications raised bring to light the discussion of an unusual clinical picture and of important repercussions for pregnant women and babies.

\section{Case report}

Female patient, 39 years old, nurse, resident in Feira de Santana, Bahia, Brazil, first-time child, affected by the IHCP at the 35th week of pregnancy, hands and soles, making sleep and routine activities difficult.

With the emergence of new symptoms, such as choluria, fecal acolism and jaundice, added to the despair of pruritus that did not stop with the use of oral antihistamine, the study nurse searched the search site for symptoms associated with pregnancy and came across 
with the possibility of being the IHCP, hitherto unknown to her and all her health colleagues that she shared the information. When she came across such intense itching on her face, associated with edema and jaundice, she contacted her obstetrician doctor who promptly directed her to seek an emergency.

When seeking help in the emergency of a private hospital, the diagnostic suspicion of IHCP was raised, and confirmed after liver function tests (GTO, Glutamic Transaminase Oxalacetic; GT, Glutamic Transaminase and Gamma GT, Gamma Glutamil Transpeptidase), renal function (urea and creatinine), bilirubins (total and fractions) and ANF, Anti-Nuclear Factor, the result of which suggested the possibility of cholestasis due to autoimmune disease.

Upon returning to the routine consultation with your obstetrician and communicating the diagnosis given by the doctor who attended to you in the emergency. Thereafter, fetal vitality was monitored weekly and ursodeoxycholic acid was prescribed for symptom relief, as well as dexamethasone for fetal maturation, with favorable liver enzyme results and symptom mitigation. After weekly follow-up with favorable results, a 39-week gestacional age caesarean section was scheduled, with a good maternal and fetal prognosis.

\section{Discussion}

The diagnosis of IHCP occurs at an unfavorable gestational age at birth, and medical management is of paramount importance for the evolution and good prognosis of the condition. ${ }^{3}$ Although IHCP symptomatology is an important factor affecting the patient's quality of life, it is benign, and is not indicative of premature termination of pregnancy as soon as its diagnosis is closed, as it does not lead to maternal liver damage of high clinical importance in relation to other liver diseases. ${ }^{2,6}$

One of the main symptoms of IHCP is severe itching that usually occurs around the third trimester, more specifically between 32 and 36 weeks, although there is more severe evidence at 18 weeks, and because it is a very common finding to allergic reactions or other dermal conditions, may go unnoticed or ignored, and prescription of antihistamines is very routine to improve this complaint. ${ }^{1,5}$ It may also present jaundice within one to four weeks of the onset of pruritus, nausea, vomiting, choluria, fecal acolism, and steatorrhea secondary to fat malabsorption.

A case study conducted with a 36-year-old and 13-week old gestational age pregnant woman revealed that she complained of severe generalized pruritus that intensified at night and interrupted her sleep, but showed no obvious skin lesions, only scratches and significant elevation of liver enzymes. ${ }^{8}$

Because the incidence of new cases is relatively low, the diagnosis of IHC is difficult, and the individualized look at each patient is indispensable, and no complaint should be dismissed or underestimated and the laboratory tests should be carefully associated and symptoms presented, since the early and assertive diagnosis IHCP is crucial to prevent the incidence of premature births, respiratory problems in the neonate, presence of meconium and intrauterine fetal death. ${ }^{5,6}$

The differential diagnosis of other liver disorders is given in the clinical context associated with laboratory tests, excluding dermatological disorders, allergic reactions and liver damage before pregnancy or caused by various other factors unrelated to the pregnancy process, such as acute, toxic, viral hepatitis or autoimmune., ${ }^{9,10}$
Once diagnosed, it is necessary to reflect on the conduct to be taken based on the thorough evaluation of the mother and fetus, which is based on laboratory results and gestational age. The classification of low, medium and high risk is given by the dosage of liver enzymes, also having the special condition that does not depend on laboratory results, being evaluated the personal history of IHCP or family and also fetal death of unknown cause. ${ }^{5,11}$

After classifying this pregnant woman, the fetus is also evaluated, and fetal monitoring is recommended every 72 hours from the 32nd gestational week. The conduct will always be taken based on the risk-benefit for the mother-fetus binomial. With good follow-up and the imminent risk of fetal death, there is no need for urgent cesarean section, and may adopt the expectant drug approach that has been shown to be effective with a good fetal prognosis. ${ }^{1,3,5}$

The approach should be taken after detailed analysis of maternal liver enzymes and fetal vitality, with medication control of damage and symptoms, so that delivery is delayed as much as possible, within the good clinical conditions of the binomial, avoiding complications and/ or fetal deaths caused, prematurity, as well as preventing intrauterine deaths caused by neglecting proper treatment due to misdiagnosis. ${ }^{2,6}$

Currently, the most used drug to combat symptoms and improve liver damage is ursodeoxycholic acid. Although its mechanism of action is not fully known, the use of this drug does not appear to have any harmful effects, as studies have shown a reduction in total serum bile acids after treatment, both in maternal serum collected through the umbilical cord and amniotic fluid, and colostrum, in addition to improving placental morphology and function. ${ }^{12}$

A study of 98 pregnant women showed that with prescribing ursodeoxycholic acid, pruritus improved in $76,5 \%$ of participants and completely disappeared before delivery in $25,5 \%$ and that after 3 weeks of treatment, liver enzyme levels decreased, more than $50 \%$ of baseline in $77,9 \%$ of patients. ${ }^{13}$

Therapy is still associated with antihistamines and corticosteroids for fetal maturation between 24 and 34 weeks, with betamethasone and dexamethasone being the most commonly used. This pregnant woman can also be referred to the nutrition service so that a hepatoprotective diet can be prescribed to reduce liver damage. ${ }^{1,5}$

Therefore, it is clear that the treatment of IHCP is controversial, as some prefer to follow the mother-fetus binomial waiting for the maximum gestation time to decrease the incidence of premature births and others seek therapies to alleviate maternal symptoms and improve fetal prognosis, however there is no proven reliable evidence of the drugs used. ${ }^{1}$

Although expectant non-drug management up to 40 weeks has been favorable in case-control studies, severe pruritus becomes a very painful disorder for its bearer, which will influence their living, sleeping, and even sentimental disorders, of anguish and despair. ${ }^{12}$

\section{Conclusion}

From the clinical case, it is important to obtain an early diagnosis of IHCP, even if complex, requiring experience and a critical and questioning look of the examiner during prenatal consultations, the probable causes for the multiple reported complaints.

Given the high morbidity of this disease for the fetus, it needs to be better debated and evaluated among professionals who attend prenatal 
of pregnant women, whether they are doctors or nurses. Therefore, it is essential to deepen the knowledge about the symptoms and risk factors of IHCP, because despite being a rare event, the fetal prognosis may be unsatisfactory if there is a failure, underestimation of the symptoms or failure in the diagnosis due to lack of knowledge about it, health professionals or because it is similar to dermatitis and/or allergic processes.

Given the difficulty in closing the diagnosis, there is also a clear need for further studies and publications in the literature on strategies to facilitate diagnosis and differentiate it from other diseases with the same symptoms to start treatment as early as possible and avoid fatal outcomes.

\section{Acknowledgments}

None.

\section{Funding}

None.

\section{Conflicts of interest}

Authors disclose no conflict of interests in publication of this study.

\section{References}

1. Souza E, Guerzet EA, Fava JL, et al. Intrahepatic cholestasis of pregnancy: scientific evidence for treatment choice. Femina. 2014;42(1):39-42.

2. Perosa M, Genzini T, Pandullo FL, et al. Acute liver failure of pregnancy: clinical experience with seven cases. Rev Bras Ginecol Obstetr. 2001;23(3):159-165.

3. Feitosa FEL, Melo DS, Monteiro MM, et al. Cholestasis of pregnancy: literature review. Femina. 2019;37(4):203-207.
4. Sepúlveda-Martíne S, Hasbun JH, Valdés ER, et al. Clinical guidelines series: Intrahepatic cholestasis of pregnancy. Rev Hosp Clin Univ Chile. 2015;26:63-72.

5. Estiú C, Frailuna MA, Dericco M, et al. Clinical Practice Guide: Gestational intrahepatic cholestasis. Rev Hosp Mat Inf Ramón Sardá. 2012;31(3):125-136

6. Silva Junior JF, Argente JS, Antunes GN, et al. Recurrent benign intrahepatic cholestasis. Revista da AMRIGS. 2008;52(3):209-211.

7. Alves GF, Varella TCN, Nogueira LSC. Dermatology and pregnancy. An Bras Dermatol. 2005;80(2):179-186.

8. Pérez González Y, Torres Rojas KL, Argote Viñals C. Intrahepatic cholestasis of pregnancy. Revista Electrónica Dr. Zoilo E. Marinello Vidaurreta. 2015;40(8).

9. Grymowicz M, Czajkowski K, Smolarczyk R. Pregnancy course in patients with intrahepatic cholestasis of pregnancy treated with very low doses of ursodeoxycholic acid. Scand J Gastroenterol. 2016;51(1):78-85.

10. Zhang Y, Hu L, Cui Y, et al. Roles of PPAR $\gamma / \mathrm{NF}-\kappa \mathrm{B}$ signaling pathway in the pathogenesis of intrahepatic cholestasis of pregnancy. PLoS One. 2014;9(1):1-11.

11. Ovadia C, Seed PR, Sklavounos A, et al. Association of adverse perinatal outcomes of intrahepatic cholestasis of pregnancy with biochemical markers: results of aggregate and individual patient data meta-analyses. Lancet. 2019; 393(10174):899-909.

12. Williamson C, Geenes V. Intrahepatic Cholestasis of Pregnancy. Obstet Gynecol. 2014;124:120-133.

13. Bacq Y, le Besco M, Lecuyer AI, et al. Ursodeoxycholic acid therapy in intrahepatic cholestasis of pregnancy: Results in real-world conditions and factors predictive of response to treatment. Dig Liver Dis. 2017;49(1):6369. 